\title{
TWO-SIDED ASYMPTOTIC BOUNDS FOR THE COMPLEXITY OF SOME CLOSED HYPERBOLIC THREE-MANIFOLDS
}

\author{
SERGEI MATVEEV, CARLO PETRONIO ${ }^{\bowtie}$ and ANDREI VESNIN
}

(Received 27 March 2006; accepted 12 December 2007)

Communicated by C. D. Hodgson

\begin{abstract}
We establish two-sided bounds for the complexity of two infinite series of closed orientable threedimensional hyperbolic manifolds, the Löbell manifolds and the Fibonacci manifolds. The manifolds of the two series are indexed by an integer $n$ and the corresponding complexity estimates are both linear in $n$.
\end{abstract}

1991 Mathematics subject classification: primary 57M27; secondary 57M50.

Keywords and phrases: 3-manifolds, complexity, hyperbolic geometry.

\section{Introduction}

If $M$ is a compact three-dimensional manifold, its complexity (introduced in Matveev $[14,15])$ is a nonnegative integer $c(M)$ that formally translates the intuitive notion of 'how complicated' $M$ is. In particular, if $M$ is closed and irreducible and different from the 3 -sphere $\mathbb{S}^{3}$, the projective 3 -space $\mathbb{R P}^{3}$, and the lens space $L(3,1)$, its complexity $c(M)$ is equal to the minimum of the number of tetrahedra over all 'singular' triangulations of $M$. (A singular triangulation of $M$ is a realization of $M$ as a union of tetrahedra with pairwise glued 2-faces.) The complexity function has many natural properties, among which is additivity under connected sum.

The task of computing the complexity $c(M)$ of a given manifold $M$ is extremely difficult. For closed $M$, the exact value is presently known only if $M$ belongs to the computer-generated tables of manifolds up to complexity 12 (see Matveev [17]). Therefore the problem of finding 'reasonably good' two-sided bounds for $c(M)$ is of

This work is the result of a collaboration among the three authors carried out in the frame of the INTAS project 'CalcoMet-GT' 03-51-3663. The first and the third authors were also supported by the Russian Fund for Fundamental Research, grants 05-01-00293 and 06-01-72014-MSCS.

(c) 2009 Australian Mathematical Society 1446-7887/2009 \$16.00 
primary importance. The first results of this kind were obtained in [18, 20], where an estimate on $c(M)$ was given in terms of certain properties of the homology groups of $M$.

In the present paper we establish two-sided bounds on the complexity of two infinite series of closed orientable three-dimensional hyperbolic manifolds, the Löbell manifolds and the Fibonacci manifolds. The upper bounds are obtained by constructing fundamental polytopes of these manifolds in hyperbolic space $\mathbb{H}^{3}$, while the lower bounds (which are only proved in an 'asymptotic' fashion, see below) are based on the calculation of their volumes. We mention here that the Löbell manifolds are constructed from polytopes that generalize the right-angled dodecahedron, and the Fibonacci manifolds are constructed from polytopes that generalize the regular icosahedron.

Before turning to the statements and proofs of our estimates, we remark that, in the class of compact 3-manifolds with nonempty boundary, exact values of complexity are currently known for two infinite families. The first one consists of manifolds whose interior finitely covers the complement of the figure-eight knot or its 'sibling' (a different manifold with the same volume) [2, 16]. The second family consists of manifolds $M$ such that $\partial M$ consists of $k \geqslant 0$ tori and a surface of genus $g \geqslant 2$, and $M$ admits an ideal triangulation with $g+k$ tetrahedra $[7,8]$.

The following results are proved in this paper.

THEOREM 1.1. If $\mathcal{L}(n)$ denotes the $n$th set of Löbell manifolds, defined below in Section 2, then for any $M \in \mathcal{L}(n)$ the complexity of $M$ satisfies the lower and upper estimates

$$
10 n \leqslant c(M) \leqslant 47 n-92,
$$

the former one being valid for sufficiently large $n$ and the latter one for all $n \geqslant 5$.

THEOREM 1.2. If $M(n)$ denotes the nth Fibonacci manifold, defined below in Section 3, then its complexity satisfies the lower and upper estimates

$$
2 n \leqslant c(M(n)) \leqslant 3 n,
$$

the former one being valid for sufficiently large $n$ and the latter one for all $n \geqslant 4$.

\section{Löbell manifolds}

In this section we obtain upper and lower bounds on manifold complexity for a certain infinite family of closed hyperbolic 3-manifolds that generalize the classical Löbell manifold. Recall that in order to give a positive answer to the question of the existence of 'Clifford-Klein space forms' (that is, closed manifolds) of constant negative curvature, Löbell [13] constructed in 1931 the first example of a closed orientable hyperbolic 3-manifold. The manifold was obtained by gluing together eight copies of the right-angled 14-faced polytope (denoted by $R(6)$ and shown in Figure 1) with upper and lower bases both being regular hexagons, and a lateral surface given by 12 pentagons, arranged similarly as in the dodecahedron. 
An algebraic approach to constructing hyperbolic 3-manifolds from eight copies of a right-angled polytope was suggested in Vesnin [23], as we will now describe. Let us fix in hyperbolic 3 -space $\mathbb{H}^{3}$ a bounded polytope $R$, namely a compact and convex set homeomorphic to the 3-disk, with boundary given by a finite union of geodesic polygons, called the faces of $R$. The notions of vertex and edge of $R$ are defined in the obvious fashion. We further assume that $R$ is right-angled, namely that all the dihedral angles along the edges of $R$ are $\pi / 2$, which easily implies that all the faces of $R$ have at least five edges and all the vertices of $R$ are trivalent. Note that, according to Andreev's theorem [1], these combinatorial conditions on an abstract polytope are actually also sufficient for its realizability as a bounded right-angled polytope in $\mathbb{H}^{3}$.

We will denote henceforth by $G$ the subgroup of $\operatorname{Isom}\left(\mathbb{H}^{3}\right)$, the isometry group of hyperbolic 3-space, generated by the reflections in the planes containing the faces of $R$. The following is an easy consequence of Poincaré's polyhedron theorem $[6,21]$.

LEMMA 2.1. The polytope $R$ is a fundamental domain for $G$, and a presentation of $G$ is given by:

(1) a generator for each face of $R$; and

(2) the relation $\rho^{2}$ for each generator $\rho$ and the relation $\left[\rho_{1}, \rho_{2}\right]$ for each pair of generators $\rho_{1}, \rho_{2}$ associated to faces sharing an edge.

This result implies that $G$ is a discrete subgroup of isometries of $\mathbb{H}^{3}$. In particular, a subgroup $K$ of $G$ will act freely on $\mathbb{H}^{3}$ if and only if it is torsion-free. Moreover for each vertex $v$ of $R$ the stabilizer $\operatorname{Stab}_{G}(v)$ of $v$ in $G$ is isomorphic to the Abelian group $\mathbb{Z}_{2} \oplus \mathbb{Z}_{2} \oplus \mathbb{Z}_{2}=\left(\mathbb{Z}_{2}\right)^{3}$ of order 8 , which we will view as a vector space over the field $\mathbb{Z}_{2}$. We next quote two lemmas proved in Vesnin [23] and derive an easy consequence.

LEMMA 2.2. If $\varphi: G \rightarrow\left(\mathbb{Z}_{2}\right)^{3}$ is an epimorphism, the following are equivalent:

(1) $\operatorname{Ker}(\varphi)$ is torsion-free; and

(2) for each vertex of $R$, if $\rho_{1}, \rho_{2}, \rho_{3}$ are the reflections in the faces of $R$ incident to the vertex, $\varphi\left(\rho_{1}\right), \varphi\left(\rho_{2}\right), \varphi\left(\rho_{3}\right)$ are linearly independent over $\mathbb{Z}_{2}$.

We consider now in $\left(\mathbb{Z}_{2}\right)^{3}$ the vectors $\alpha=(1,0,0), \beta=(0,1,0), \gamma=(0,0,1)$ and $\delta=\alpha+\beta+\gamma=(1,1,1)$, and we note that any three of them are linearly independent.

LEMMA 2.3. Let $\varphi: G \rightarrow\left(\mathbb{Z}_{2}\right)^{3}$ be an epimorphism that maps each of the generating reflections of $G$ to one of the elements $\alpha, \beta, \gamma, \delta$. Then $\operatorname{Ker}(\varphi)$ is a subgroup of Isom $^{+}\left(\mathbb{H}^{3}\right)$, the group of orientation-preserving isometries of hyperbolic 3-space.

PROPOSITION 2.4. If an epimorphism $\varphi: G \rightarrow\left(\mathbb{Z}_{2}\right)^{3}$ satisfies condition (2) of Lemma 2.2 and the hypothesis of Lemma 2.3, then the quotient $M=\mathbb{H}^{3} / \operatorname{Ker}(\varphi)$ is a closed orientable hyperbolic 3-manifold.

PROOF. Lemma 2.2 implies that $\operatorname{Ker}(\varphi)$ is torsion-free, so the quotient is a hyperbolic 3-manifold $M$ without boundary, and Lemma 2.3 implies that $M$ is orientable. Since 

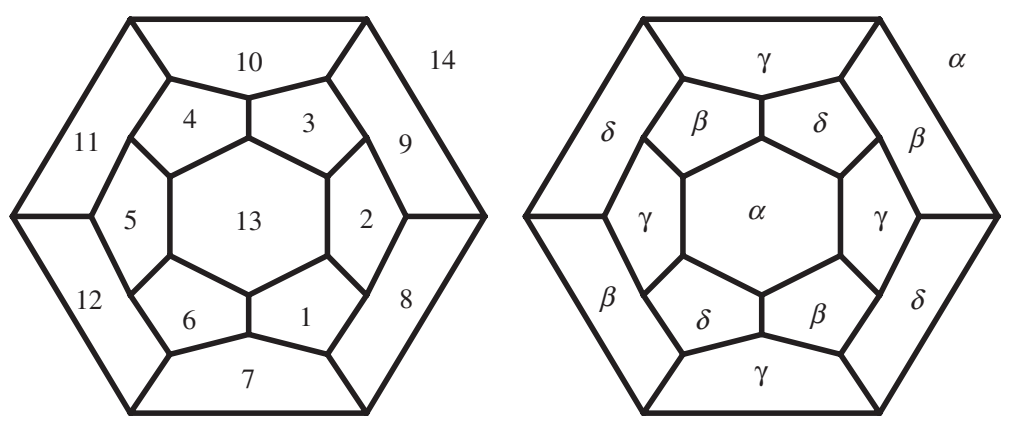

FIGURE 1. The polytope $R(6)$ and a coloring of its faces.

$\operatorname{Ker}(\varphi)$ has index 8 in $G$ and $R$ is a fundamental domain for $G$, a fundamental domain for $\operatorname{Ker}(\varphi)$ is given by $\bigcup_{i=1}^{8} g_{i}(R)$ where $\left\{g_{i} \mid 1 \leqslant i \leqslant 8\right\}$ is a set of representatives of $G / \operatorname{Ker}(\varphi)$. Such a fundamental domain is compact, so $M$ is compact, whence closed.

If we now describe a homomorphism of $G$ by labeling each face of $R$ by the image of the reflection in the plane containing that face, a map $\varphi$ as in Proposition 2.4 gives an $\{\alpha, \beta, \gamma, \delta\}$ coloring of the faces of $R$ with the usual condition that adjacent faces should have different colors. On the other hand, Lemma 2.1 implies the converse, namely that any such coloring gives a map $\varphi$ as in Proposition 2.4, and hence a closed orientable hyperbolic manifold. As an example, the classical Löbell manifold [13] is obtained from the polytope $R(6)$ described above and shown on the left of Figure 1 using the coloring shown on the right in the same figure.

We generalize this example by considering for each $n \geqslant 5$ the right-angled polytope $R(n)$ in $\mathbb{H}^{3}$ with $(2 n+2)$ faces, two of which (viewed as the upper and lower bases) are regular $n$-gons, while the lateral surface is given by $2 n$ pentagons, arranged as one easily imagines. Note that $R(5)$ is the right-angled dodecahedron. As in Figure 1 for $R(6)$, we number the faces of $R(n)$ so that:

(i) the upper and lower bases have numbers $2 n+1$ and $2 n+2$, respectively;

(ii) the pentagons adjacent to the upper base are cyclically numbered $1, \ldots, n$; and

(iii) the pentagons adjacent to the lower base are cyclically numbered $n+1, \ldots, 2 n$ in the same sense as the previous ones, with pentagon $n+1$ adjacent to pentagons 1 and $n$.

Now define $g_{i} \in \operatorname{Isom}\left(\mathbb{H}^{3}\right)$ as the reflection in the plane containing the $i$ th face of $R(n)$, and $G(n)$ as the group generated by $\left\{g_{i}\right\}_{i=1}^{2 n+2}$. According to Lemma 2.1 a presentation of $G(n)$ is obtained by adding the relations: 


$$
\begin{array}{ll}
g_{i}^{2}, & i=1, \ldots, 2 n+2 ; \\
{\left[g_{2 n+1}, g_{i}\right],} & i=1, \ldots, n ; \\
{\left[g_{2 n+2}, g_{n+i}\right],} & i=1, \ldots, n ; \\
{\left[g_{i}, g_{i+1}\right],} & i=1, \ldots, 2 n-1 ; \\
{\left[g_{1}, g_{n}\right],} & \\
{\left[g_{i}, g_{n+i}\right],} & i=1, \ldots, n ; \\
{\left[g_{n+1}, g_{2 n}\right],} & \\
{\left[g_{i}, g_{n+1+i}\right],} & i=1, \ldots, n-1 .
\end{array}
$$

We now define the class of Löbell manifolds of order $n$ as follows:

$$
\begin{array}{r}
\mathcal{L}(n)=\left\{\mathbb{H}^{3} / \operatorname{Ker}(\varphi) \mid \varphi: G(n) \rightarrow\left(\mathbb{Z}_{2}\right)^{3} \text { epimorphism },\right. \\
\left.\operatorname{Ker}(\varphi)<\operatorname{Isom}^{+}\left(\mathbb{H}^{3}\right), \operatorname{Ker}(\varphi) \text { is torsion-free }\right\}
\end{array}
$$

Each element of $\mathcal{L}(n)$ is a closed orientable hyperbolic 3-manifold with volume equal to eight times the volume of $R(n)$. According to the above discussion (and the four-color theorem [3]) the set $\mathcal{L}(n)$ is nonempty for all $n \geqslant 5$. The classical Löbell manifold constructed in [13] and described above belongs to $\mathcal{L}(6)$. We now provide an upper bound for the complexity of the elements of $\mathcal{L}(n)$.

Proposition 2.5. For all $n \geqslant 5$ and $M \in \mathcal{L}(n)$, we have

$$
c(M) \leqslant 47 n-92 .
$$

PROOF. Let $M$ be defined by a homomorphism $\varphi: G(n) \rightarrow\left(\mathbb{Z}_{2}\right)^{3}$. By our construction $M$ is built by gluing together in pairs the faces of eight copies of $R(n)$. More precisely, let us pick a vertex $v$ of $R(n)$ at the corner of three faces $A, B, C$, and let us denote by $a, b, c$ the reflections in $A, B, C$. Then $S=\langle a, b, c\rangle$ is the stabilizer of $v$ in $G(n)$. It is an Abelian group isomorphic to $\left(\mathbb{Z}_{2}\right)^{3}$, it is a set of representatives of $G(n) / \operatorname{Ker}(\varphi)$, and

$$
Q(n)=\bigcup_{s \in S} s(R(n))
$$

is a fundamental domain for the action of $\operatorname{Ker}(\varphi)$ on $\mathbb{H}^{3}$ giving $M$ as a quotient.

Up to isometry $R(n)$ has two inequivalent types of vertices (unless $n=5$ ). Therefore, to analyze the structure of $Q(n)$ we must make a definite choice of $v$. We select $v$ as a vertex of one of the $n$-gons and redraw $R(n)$ as the prism shown in Figure 2 (which refers to the case $n=8$ ). Hence $Q(n)$ is obtained by taking the images of this prism under the reflections in the faces containing $v$. In particular, from a combinatorial viewpoint, $Q(n)$ has $16(n-1)$ faces that are pentagons and eight faces that are $n$-gons. The geometric picture is different, however, because some adjacent pentagons are coplanar and hence give rise to faces of $Q(n)$ having more than five edges. More precisely, the following happens: 


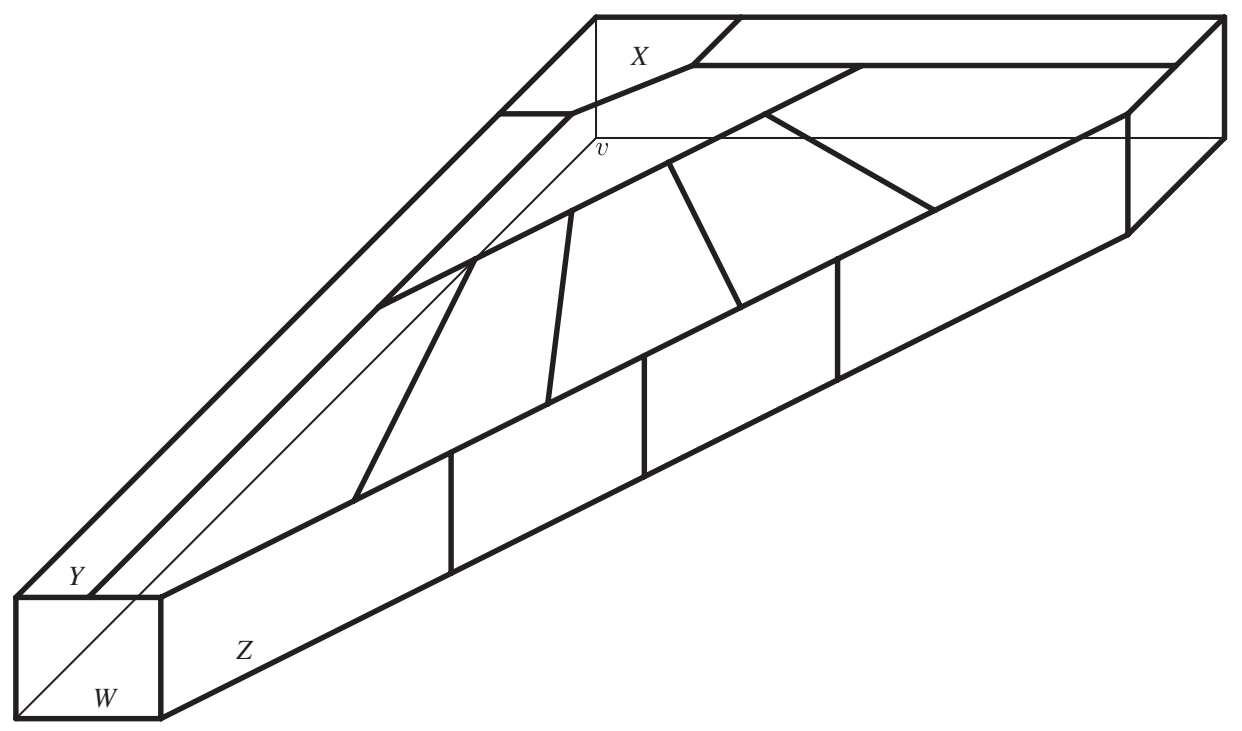

FIGURE 2. The polytope $R(n)$ for $n=8$.

(1) the pentagon $X$ of the prism together with three reflected copies of $X$ gives rise to a 'horizontal' octagon;

(2) the pentagon $Y$ of the prism together with one reflected copy of $Y$ gives rise to a 'horizontal' hexagon;

(3) the pentagon $W$ of the prism together with three reflected copies of $W$ gives rise to a 'vertical' octagon; and

(4) the pentagon $Z$ of the prism together with one reflected copy of $Z$ gives rise to a 'vertical' hexagon.

The number of times these phenomena (1), (2), (3) and (4) globally happen on $\partial Q(n)$ is respectively $2,4,8$ and $4(n-4)$. Therefore $Q(n)$ has the following number of faces:

(i) $n$-gons, 8 ;

(ii) octagons, $2+4=6$;

(iii) hexagons, $8+4(n-4)=4(n-2)$; and

(iv) pentagons, $16(n-1)-4 \times 6-2 \times 4(n-2)=8(n-3)$.

LEMMA 2.6. $\operatorname{Ker}(\varphi)$ acts on $Q(n)$ by gluing together in pairs the faces just described.

PROOF. The pentagonal faces of $Q(n)$, before being merged into hexagons and octagons, have the form $s(F)$, where $s$ varies in $S$ and $F$ varies in the faces of $R(n)$ different from $A, B, C$. Given such $s$ and $F$, there exist unique $s^{\prime} \in S$ and $k \in \operatorname{Ker}(\varphi)$ such that $s^{\prime}=k \cdot s \cdot \rho_{F}$, where $\rho_{F}$ is the reflection in the plane containing $F$; and under these assumptions the pentagon to which $s(F)$ is glued is $s^{\prime}(F)$ with gluing map $k: s(F) \rightarrow s^{\prime}(F)$. 
Now suppose that $s_{1}\left(F_{1}\right)$ and $s_{2}\left(F_{2}\right)$ are pentagons lying in the same plane, whence merged into some hexagonal or octagonal face of $Q(n)$. This means that $F_{1}=F_{2}=: F$ and $s_{2}=s_{1} \cdot s$ with $s \cdot \rho_{F}=\rho_{F} \cdot s$. Let $s_{1}^{\prime}, s_{2}^{\prime} \in S$ and $k_{1}, k_{2} \in \operatorname{Ker}(\varphi)$ be such that

$$
s_{1}^{\prime}=k_{1} \cdot s_{1} \cdot \rho_{F}, \quad s_{2}^{\prime}=k_{2} \cdot s_{2} \cdot \rho_{F} .
$$

Now

$$
k_{1} \cdot s_{2} \cdot \rho_{F}=k_{1} \cdot s_{1} \cdot s \cdot \rho_{F}=k_{1} \cdot s_{1} \cdot \rho_{F} \cdot s=s_{1}^{\prime} \cdot s,
$$

and this element belongs to $S$. Therefore $k_{2}=k_{1}=: k$ and $s_{2}^{\prime}=s_{1} \cdot s$, which means that the same element $k$ of $\operatorname{Ker}(\varphi)$ glues $s_{1}(F)$ to $s_{1}^{\prime}(F)$ and $s_{2}(F)$ to $s_{2}^{\prime}(F)$. In particular, $s_{1}^{\prime}(F)$ and $s_{2}^{\prime}(F)$ are coplanar as $s_{1}(F)$ and $s_{2}(F)$ are, so they belong to the same merged face of $Q(n)$, which implies the conclusion.

To finish with the proof of Proposition 2.5, we pick a vertex $x$ of $Q(n)$ at the corner of an $n$-gon, a hexagon and an octagon. Note that no two of these faces are paired together by the action of $\operatorname{Ker}(\varphi)$ : this is obvious for $n \neq 6,8$, and for $n=6,8$ it follows from the fact that the pairing of hexagons and octagons is induced by a pairing of the pentagons they are the merging of. Therefore we can subdivide the $n$-gon, octagon and hexagon incident to $x$ by taking diagonals from $x$, and we can extend this subdivision to a triangulation of $\partial Q(n)$ that is coherent under the action of $\operatorname{Ker}(\varphi)$. This implies that by taking cones from $x$ we can subdivide $Q(n)$ into as many tetrahedra as there are triangles not containing $x$, getting a singular triangulation of $M$ in the quotient. We are only left to compute the number of triangles on $\partial Q(n)$ not incident to $x$ :

$$
\begin{aligned}
& (8-1)(n-2)+(6-1)(8-2)+(4(n-2)-1)(6-2)+8(n-3)(5-2) \\
& \quad=47 n-92 .
\end{aligned}
$$

The proposition is proved.

To give lower complexity estimates, both for Löbell manifolds and for the Fibonacci manifolds considered in Section 3, we will employ the hyperbolic volume. As a matter of fact, there is a general inequality, basically due to Thurston [22], between complexity and volume. We include a proof for the sake of completeness (see also Remark 2.8). Here and very often in the sequel we will denote by $v_{3}=1.014 \ldots$ the volume of the regular ideal tetrahedron in $\mathbb{H}^{3}$ (see $\left.[4,22]\right)$.

PROPOSITION 2.7. If $M$ is a closed orientable hyperbolic manifold then

$$
c(M)>\frac{\operatorname{vol}(M)}{v_{3}} .
$$

Proof. Denote $c(M)$ by $k$. Since $M$ is irreducible and not one of the exceptional manifolds $\mathbb{S}^{3}, \mathbb{R P}^{3}$, and $L(3,1)$, there exists a realization of $M$ as a gluing of $k$ tetrahedra. Denoting by $\Delta$ the abstract tetrahedron, this realization induces continuous 
maps $\sigma_{i}: \Delta \rightarrow M$ for $i=1, \ldots, k$ describing how the tetrahedra appear in $M$ after the gluing. Note that each $\sigma_{i}$ is injective on the interior of $\Delta$ but perhaps not on the boundary. Since the gluings used to pair the faces of the tetrahedra in the construction of $M$ are simplicial, we see that $\sum_{i=1}^{k} \sigma_{i}$ is a singular 3-cycle, which of course represents the fundamental class $[M] \in \mathrm{H}_{3}(M ; \mathbb{Z})$.

Let us now consider the universal covering $\mathbb{H}^{3} \rightarrow M$. Since $\Delta$ is simply connected, we can lift $\sigma_{i}$ to a map $\widetilde{\sigma}_{i}: \Delta \rightarrow \mathbb{H}^{3}$. We now denote by $\tilde{\tau}_{i}: \Delta \rightarrow \mathbb{H}^{3}$ the simplicial map that agrees with $\widetilde{\sigma}_{i}$ on the vertices, where geodesic convex combinations are used in $\mathbb{H}^{3}$ to define the notion of 'simplicial'. Let $\tau_{i}: \Delta \rightarrow M$ be the composition of $\tilde{\tau}_{i}$ with the projection $\mathbb{H}^{3} \rightarrow M$. One can easily see that $\sum_{i=1}^{k} \tau_{i}$ is again a singular 3cycle in $M$. Using this fact and taking convex combinations in $\mathbb{H}^{3}$ one can actually see that the cycles $\sum_{i=1}^{k} \sigma_{i}$ and $\sum_{i=1}^{k} \tau_{i}$ are homotopic. In particular, since the first cycle represents $[M]$, the latter also does, which implies that $\bigcup_{i=1}^{k} \tau_{i}(\Delta)$ is equal to $M$, otherwise $\sum_{i=1}^{k} \tau_{i}$ would be homotopic to a map with two-dimensional image.

Let us now note that $\tilde{\tau}_{i}(\Delta)$ is a compact geodesic tetrahedron in $\mathbb{H}^{3}$, and therefore its volume is less than $v_{3}$ (see [4]). Moreover the volume of $\tau_{i}(\Delta)$ is at most equal to the volume of $\tilde{\tau}_{i}(\Delta)$, because the projection $\mathbb{H}^{3} \rightarrow M$ is a local isometry, and the volume of $M$ is at most the sum of the volumes of the $\tau_{i}(\Delta)$, because we have shown above that $M$ is covered by the $\tau_{i}(\Delta)$. This concludes the proof.

REMARK 2.8. The statement of [22, Corollary 6.1.7] would imply the nonstrict inequality $c(M) \geqslant \operatorname{vol}(M) / v_{3}$, but our proof of the strict inequality closely mimics the arguments given by Thurston therein.

Getting back to our Löbell manifolds, in order to apply Proposition 2.7 let us denote by $\ell_{n}$ the common value of $\operatorname{vol}(M)$ as $M$ varies in $\mathcal{L}(n)$, and recall the definition of the Lobachevskii function [4, 21, 22],

$$
\Lambda(x)=-\int_{0}^{x} \log |2 \sin (t)| d t .
$$

The following theorem was established in Vesnin [24].

THEOREM 2.9. For all $n \geqslant 5$,

$$
\ell_{n}=4 n\left(2 \Lambda\left(\theta_{n}\right)+\Lambda\left(\theta_{n}+\frac{\pi}{n}\right)+\Lambda\left(\theta_{n}-\frac{\pi}{n}\right)+\Lambda\left(\frac{\pi}{2}-2 \theta_{n}\right)\right),
$$

where

$$
\theta_{n}=\frac{\pi}{2}-\arccos \left(\frac{1}{2 \cos (\pi / n)}\right)
$$

This theorem implies that the volume of the classical Löbell manifold is equal to $48.184368 \ldots$ In addition, it allows us to determine the asymptotic behavior of $\ell_{n}$ as $n$ tends to infinity. 
PROPOSITION 2.10. The following inequalities hold for sufficiently large $n$ :

$$
10 n-\frac{68}{n}<\frac{\ell_{n}}{v_{3}}<10 n \text {. }
$$

Proof. Setting

$$
\begin{aligned}
& \theta(t)=\frac{\pi}{2}-\arccos \left(\frac{1}{2 \cos t}\right), \\
& f(t)=2 \Lambda(\theta(t))+\Lambda(\theta(t)+t)+\Lambda(\theta(t)-t)+\Lambda(\pi / 2-2 \theta(t)),
\end{aligned}
$$

we see that $\ell_{n}=4 n \cdot f(\pi / n)$. Now $\theta$ is a smooth function at 0 and $\theta(0)=\pi / 6$ and $\theta^{\prime}(0)=0$, while $\Lambda$ is a smooth function at $\pi / 6$ and

$$
\Lambda(\pi / 6)=v_{3} / 2, \quad \Lambda^{\prime}(\pi / 6)=0, \quad \Lambda^{\prime \prime}(\pi / 6)=-\sqrt{3}
$$

(see $[4,22]$ for the first equality; the subsequent ones are obtained by direct calculation). Using these facts it is very easy to show that

$$
f(0)=\frac{5}{2} v_{3}, \quad f^{\prime}(0)=0, \quad f^{\prime \prime}(0)=-2 \sqrt{3} .
$$

Taylor's formula then implies that, for $n \gg 0$,

$$
\frac{\ell_{n}}{v_{3}}=10 n-\frac{4 \sqrt{3} \pi^{2}}{v_{3}} \frac{1}{n}+\mathrm{o}(1 / n),
$$

and the coefficient of $1 / n$ is computed as $67.43 \ldots$, which easily implies the conclusion.

The right-hand estimate in Proposition 2.10 is not strictly necessary for our purposes, but we thought it worth including it to show that no lower estimate for $\ell_{n} / v_{3}$ better than $10 n-68 / n$ can be given, at least as far as the coefficient of $n$ is concerned. In the same spirit, we will now show that the inequality holds for all $n$ and not only asymptotically.

PROPOSITION 2.11. For all $n \geqslant 5$, the following inequality holds:

$$
\frac{\ell_{n}}{v_{3}}<10 n
$$

Proof. Proposition 2.10 implies that

$$
\lim _{n \rightarrow \infty} \frac{\ell_{n}}{n}=10 v_{3},
$$

so it will be sufficient to prove that $n \mapsto \ell_{n} / n$ is an increasing function. Now $\ell_{n}=8 \operatorname{vol}(R(n))$ and one can see that $R(n)$ decomposes into $2 n$ copies of a hexahedron $P(n)$ as shown in Figure 3, with each dihedral angle equal to $\pi / 2$ except 

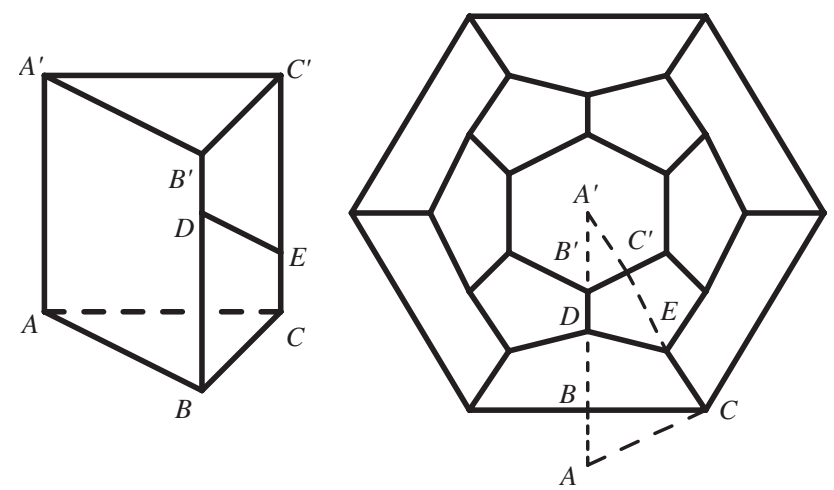

FIgURE 3. The hexahedron $P(n)$ and its position in $R(n)$ for $n=6$.

along $A A^{\prime}$, where it is $\pi / n$, and along $B^{\prime} D$ and $C E$, where it is $\pi / 4$. (As a matter of fact, this observation, together with previous work of Kellerhals [11], was precisely the basis of the computation of $\ell_{n}$ in Vesnin [24]). Therefore $\ell_{n} / n=16 \operatorname{vol}(P(n))$, so it is sufficient to prove that $n \mapsto \operatorname{vol}(P(n))$ is an increasing function, which is a direct consequence of Schläfli's variation formula [12, p. 512], since in $P(n)$ all dihedral angles are independent of $n$, except for one that decreases as $n$ increases.

We can now estimate the complexity of Löbell manifolds from the following result.

COROLlary 2.12. For sufficiently large $n$ and $M \in \mathcal{L}(n)$, we have $c(M) \geqslant 10 n$.

ProOf. Propositions 2.7 and 2.10 imply that $c(M)>10 n-(68 / n)$ for $n \gg 0$. However, $c(M)$ is an integer, and the conclusion easily follows.

Theorem 1.1 of the Introduction is now a direct consequence of Proposition 2.5 and Corollary 2.12.

\section{Fibonacci manifolds}

In this section we consider the compact orientable hyperbolic 3-manifolds whose fundamental groups are the Fibonacci groups, introduced by Conway in [5]. There is one such group $F(2, n)$ for each $n \geqslant 3$, and a presentation of it is given by

$$
F(2, n)=\left\langle x_{1}, x_{2}, \ldots, x_{n}: x_{i} x_{i+1} x_{i+2}^{-1}, i=1, \ldots, n\right\rangle,
$$

where indices are understood modulo $n$. It was shown in [9] that for each $n \geqslant 4$ the group $F(2,2 n)$ is isomorphic to a discrete cocompact subgroup of $\operatorname{Isom}^{+}\left(\mathbb{H}^{3}\right)$, the group of orientation-preserving isometries of hyperbolic 3-space. We will need below to refer to several details of the construction of [9], so we recall it here.

We fix $n \geqslant 4$ and we first define the order- $n$ antiprism $A(n)$ as the polytope whose boundary is constructed as follows: 


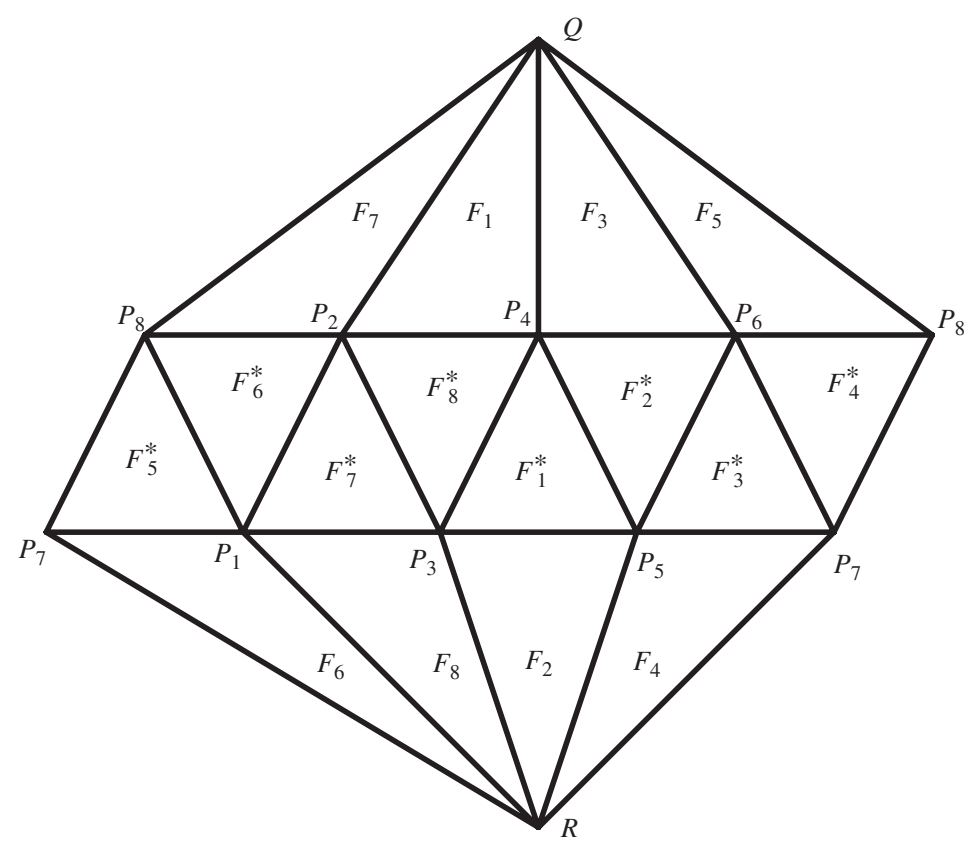

FIGURE 4. The polytope $Y(4)$.

(i) take $2 n$ triangles and two polygons with $n$ edges;

(ii) attach a different triangle to each edge of each of the two $n$-polygons; and

(iii) glue together the objects thus obtained by matching the free edges of the triangles (there are two circles consisting of $2 n$ edges to glue together, so there is essentially only one way to do so).

Now we define $Y(n)$ as the polytope obtained from $A(n)$ by attaching an $n$-pyramid to each of the bases. In particular, $Y(5)$ is the icosahedron. We remark that in general $Y(n)$ has $2 n+2$ vertices, $6 n$ edges, and $4 n$ triangular faces, and we denote the vertices by $Q, R, P_{1}, \ldots, P_{2 n}$ and the faces by $F_{1}, \ldots, F_{n}$, and $F_{1}^{*}, \ldots, F_{n}^{*}$, as shown in Figure 4 for $n=4$.

We now define a face-pairing on $Y(n)$ under which each face $F_{i}$ is glued to the face $F_{i}^{*}$ via a simplicial homeomorphism $s_{i}: F_{i} \longrightarrow F_{i}^{*}$. We specify the action of $s_{i}$ by describing it on the vertices. Namely, for odd $i$ we choose $s_{i}$ so that

$$
s_{i}: Q P_{i+1} P_{i+3} \longrightarrow P_{i+2} P_{i+3} P_{i+4},
$$

whereas for even $i$ we choose it so that

$$
s_{i}: R P_{i+1} P_{i+3} \longrightarrow P_{i+2} P_{i+3} P_{i+4},
$$

where all indices are taken modulo $2 n$. Note that if we choose an orientation of $Y(n)$ and orient the $F_{i}$ and $F_{i}^{*}$ accordingly, all the $s_{i}$ are orientation- 
reversing homeomorphisms. This implies that the quotient of $Y(n)$ under the facepairing is a manifold except perhaps at the vertices, and that the projection restricted to each open edge of $Y(n)$ is injective. In particular, we can describe how the various edges of $Y(n)$ are cyclically arranged around an edge in the quotient. These cycles are actually easy to describe: for odd $i$,

$$
Q P_{i+1} \stackrel{s_{i}}{\longrightarrow} P_{i+2} P_{i+3} \stackrel{s_{i-1}^{-1}}{\longrightarrow} P_{i} P_{i+2} \stackrel{s_{i-2}^{-1}}{\longrightarrow} Q P_{i+1},
$$

and for even $i$,

$$
R P_{i+1} \stackrel{s_{i}}{\longrightarrow} P_{i+2} P_{i+3} \stackrel{s_{i-1}^{-1}}{\longrightarrow} P_{i} P_{i+2} \stackrel{s_{i-2}^{-1}}{\longrightarrow} R P_{i+1} .
$$

It was shown in [9] that $Y(n)$ can be realized in a unique way (up to isometry) as a compact polytope in hyperbolic space $\mathbb{H}^{3}$ so that:

(i) each of the faces of $Y(n)$ is an equilateral triangle;

(ii) the sums of the dihedral angles corresponding to each of the cycles as in (3.1) or (3.2) is $2 \pi$; and

(iii) $Y(n)$ has a cyclic symmetry of order $n$ with axis $Q R$ and an orientation-reversing symmetry which permutes $Q$ and $R$.

We will henceforth identify $Y(n)$ with such a realization in $\mathbb{H}^{3}$. Since all the faces of $Y(n)$ are congruent, each face-pairing $s_{i}$ can be realized in a unique fashion as an orientation-preserving isometry of $\mathbb{H}^{3}$, and we will denote this isometry also by $s_{i}$. The condition that the total dihedral angle around the edge cycles (3.1) and (3.2) is $2 \pi$ easily implies that

$$
s_{i} s_{i+1}=s_{i+2}, \quad i=1, \ldots, 2 n,
$$

where indices are understood modulo $2 n$. More precisely, Poincaré's polyhedron theorem $[6,21]$ implies that:

(i) the subgroup of $\operatorname{Isom}^{+}\left(\mathbb{H}^{3}\right)$ generated by the $s_{i}$ is isomorphic to $F(2,2 n)$;

(ii) this group is discrete and torsion-free, and $Y(n)$ is a fundamental domain for its action on $\mathbb{H}^{3}$; and

(iii) the quotient of $\mathbb{H}^{3}$ under this action is a 3-manifold.

From now on we will denote by $M(n)$ the closed hyperbolic 3-manifold thus obtained, and call it the $n$th Fibonacci manifold. It was remarked in [10] that $M(n)$ is the $n$-fold cyclic covering of $\mathbb{S}^{3}$ branched over the figure-eight knot $4_{1}$.

Proposition 3.1. For $n \geqslant 4$, we have $c(M(n)) \leqslant 3 n$.

PROOF. For each triangular face of $Y(n)$ not containing $Q$, we can construct the tetrahedron with vertex at $Q$ and basis at that face. This gives a decomposition of $Y(n)$ into $3 n$ tetrahedra, namely a singular triangulation of $M(n)$ with $3 n$ tetrahedra, whence the conclusion.

To estimate the complexity of $M(n)$, we use the following formula for its volume established in [19]. 
THEOREM 3.2. For $n \geqslant 4$,

$$
\operatorname{vol}(M(n))=2 n\left(\Lambda\left(a_{n}+\pi / n\right)+\Lambda\left(a_{n}-\pi / n\right)\right),
$$

where $a_{n}=(1 / 2) \arccos (\cos (2 \pi / n)-1 / 2)$.

This result allows us to determine the asymptotic behavior of the volume of the Fibonacci manifold $M(n)$.

PROPOSITION 3.3. The following inequalities hold for sufficiently large n:

$$
2 n-\frac{34}{n}<\frac{\operatorname{vol}(M(n))}{v_{3}}<2 n .
$$

PROOF. The proof is very similar to that of Proposition 2.10. Setting

$$
\begin{gathered}
a(t)=(1 / 2) \arccos (\cos (2 t)-1 / 2), \\
f(t)=\Lambda(a(t)+t)+\Lambda(a(t)-t),
\end{gathered}
$$

we see that $\operatorname{vol}(M(n))=2 n f(\pi / n)$. Again $a(0)=\pi / 6$ and $a^{\prime}(0)=0$, whence, using the values of $\Lambda, \Lambda^{\prime}, \Lambda^{\prime \prime}$ at $\pi / 6$, we get

$$
f(0)=v_{3}, \quad f^{\prime}(0)=0, \quad f^{\prime \prime}(0)=-2 \sqrt{3} .
$$

Therefore

$$
\frac{\operatorname{vol}(M(n))}{v_{3}}=2 n-\frac{2 \sqrt{3} \pi^{2}}{v_{3}} \frac{1}{n}+\mathrm{o}(1 / n),
$$

and the coefficient of $1 / n$ is $33.71 \ldots$, which easily implies the conclusion.

Analogously to Proposition 2.11 we are able to show that the right-hand side estimate in Proposition 3.3 is always true, not only asymptotically.

PROPOSITION 3.4. For all $n \geq 4$, the following inequality holds:

$$
\frac{\operatorname{vol}(M(n))}{v_{3}}<2 n .
$$

Proof. Proposition 3.3 implies that

$$
\lim _{n \rightarrow \infty} \frac{\operatorname{vol}(M(n))}{n}=2 v_{3},
$$

so it is sufficient to prove that $n \mapsto \operatorname{vol}(M(n)) / n$ is an increasing function. To this end we recall a result to be found in [10], namely that $M(n)$ is the $n$-fold cyclic covering of $\mathbb{S}^{3}$ branched over the figure-eight knot $4_{1}$. Let now us denote by $4_{1}(n)$ the orbifold with underlying space $\mathbb{S}^{3}$ and singular set $4_{1}$ with cone angle $\pi / n$. Since $4_{1}(n)$ is hyperbolic and $\operatorname{vol}\left(4_{1}(n)\right)=\operatorname{vol}(M(n)) / n$, it is then sufficient to demonstrate that $n \mapsto \operatorname{vol}\left(4_{1}(n)\right)$ is an increasing function. But this fact is a direct consequence of Schläfli's variation formula [12, p. 512], since the cone angle decreases as $n$ increases. 
Using again the fact that the complexity of a manifold is always an integer, as in the proof of Corollary 2.12, this result together with Proposition 2.7 implies the following result.

COROLlary 3.5. For sufficiently large $n$, we have $c(M(n)) \geqslant 2 n$.

Theorem 1.2 of the Introduction is now a direct consequence of Proposition 3.1 and Corollary 3.5 .

\section{Acknowledgements}

The authors thank Ekaterina Pervova for her help and the referee for useful comments.

\section{References}

[1] E. M. Andreev, 'On convex polyhedra in Lobachevskii spaces', Math. USSR Sb. 10 (1970), 413-440.

[2] S. Anisov, 'Exact values of complexity for an infinite number of 3-manifolds', Mosc. Math. J. 5 (2005), 305-310.

[3] K. Appel and W. Haken, Every Planar Map is Four Colorable, Contemporary Mathematics, 98 (American Mathematical Society, Providence, RI, 1989).

[4] R. Benedetti and C. Petronio, Lectures on Hyperbolic Geometry (Springer, Berlin, 1992).

[5] J. Conway, 'Advanced problem 5327', Amer. Math. Monthly 72 (1965), 915.

[6] D. B. A. Epstein and C. Petronio, 'An exposition of Poincaré's polyhedron theorem', Enseign. Math. 40 (1994), 113-170.

[7] R. Frigerio, B. Martelli and C. Petronio, 'Complexity and Heegaard genus of an infinite class of compact 3-manifolds', Pacific J. Math. 210 (2003), 283-297.

[8] - 'Dehn filling of cusped hyperbolic 3-manifolds with geodesic boundary', J. Differential Geom. 64 (2003), 425-455.

[9] H. Helling, A. C. Kim and J. L. Mennicke, 'A geometric study of Fibonacci groups', J. Lie Theory 8 (1999), 1-23.

[10] H. M. Hilden, M. T. Lozano and J. M. Montesinos-Amilibia, 'The arithmeticity of the figure eight knot orbifolds', Topology '90, Columbus, OH, 1990 (de Gruyter, Berlin, 1992), pp. 169-183.

[11] R. Kellerhals, 'On the volume of hyperbolic polyhedra', Math. Ann. 285 (1989), 541-569.

[12] S. Kojima, 'Deformation of hyperbolic 3-cone-manifolds', J. Differential Geom. 48 (1999), 469-516.

[13] F. Löbell, 'Beispiele geschlossene dreidimensionaler Clifford-Kleinischer Räume negative Krümmung', Ber. Verh. Sächs. Akad. Lpz., Math. Phys. Kl. 83 (1931), 168-174.

[14] S. V. Matveev, 'The complexity of three-dimensional manifolds and their enumeration in the order of increasing complexity', Soviet Math. Dokl. 38 (1989), 75-78.

[15] - 'Complexity theory of three-dimensional manifolds', Acta Appl. Math. 19 (1990), 101-130.

[16] - Algorithmic Topology and Classification of 3-Manifolds, ACM Monographs, 9 (Springer, New York, 2003).

[17] _ 'Recognition and tabulation of 3-manifolds', Dokl. Akad. Nauk 400 (2005), 26-28 (in Russian).

[18] S. V. Matveev and E. Pervova, 'Lower bounds for the complexity of three-dimensional manifolds', Dokl. Math. 63 (2001), 314-315.

[19] A. D. Mednykh and A. Yu. Vesnin, 'Hyperbolic volumes of Fibonacci manifolds', Siberian Math. J. 36 (1995), 235-245. 
[20] E. Pervova and C. Petronio, 'Complexity and T-invariant of Abelian and Milnor groups, and complexity of 3-manifolds", Math. Nachr. 281 (2008), 1182-1195.

[21] O. V. Schwartsman and E. B. Vinberg, 'Discrete groups of motions of spaces of constant curvature', in: Geometry II: Spaces of Constant Curvature, Encyclopaedia of Mathematical Sciences, 29 (ed. E. B. Vinberg) (Springer, Berlin, 1993), pp. 139-248.

[22] W. P. Thurston, 'The Geometry and Topology of Three-Manifolds', Mimeographed notes, Princeton, NJ, 1979.

[23] A. Yu. Vesnin, 'Three-dimensional hyperbolic manifolds of Löbell type', Siberian Math. J. 28 (1987), 731-734.

[24] _ _ 'Volumes of hyperbolic Löbell manifolds', Math. Notes 64 (1998), 17-23.

SERGEI MATVEEV, Chelyabinsk State University, Chelyabinsk 454021, Russia e-mail: matveev@csu.ru

CARLO PETRONIO, Dipartimento di Matematica Applicata, Università di Pisa, Via Filippo Buonarroti 1C, 56127 Pisa, Italy

e-mail: petronio@dm.unipi.it

ANDREI VESNIN, Sobolev Institute of Mathematics, Novosibirsk 630090, Russia e-mail: vesnin@math.nsc.ru 